\title{
Article
}

\section{A Novel Series of Thiazoles and 1,3,4-Thiadiazoles Bearing Thiazole Moiety as Anticancer Agents: Synthesis, Spectral Studies, Biological Evaluation and Structure Activity relationship}

\author{
Sobhi M. Gomha ${ }^{1}$, Nabila A. Kheder ${ }^{1,2}$, Mohamad R. Abdelaziz ${ }^{3}$ and Yahia Nasser Mabkhot ${ }^{4, *}$ \\ 1 Department of Chemistry, Faculty of Science, Cairo University, Giza 12613, Egypt; \\ s.m.gomha@gmail.com (S.M.G.); Nabila.abdelshafy@gmail.com (N.A.K.) \\ 2 Department of Pharmaceutical Chemistry, Faculty of Pharmacy, King Khalid University, \\ Abha 61441, Saudi Arabia \\ 3 Department of pharmaceutical Chemistry, Faculty of Pharmacy, MIU University, Cairo, Egypt; \\ Mohamedvac_75@yahoo.com \\ 4 Department of Chemistry, College of Science, King Saud University, P. O. Box 2455, \\ Riyadh-11451, Saudi Arabia \\ * Correspondence: yahia@ksu.edu.sa; Tel.: +966-11-467-5898; Fax: +966-11-467-5992
}

\begin{abstract}
A novel series of thiazole based-1,3,4-thiadiazoles were designed and prepared via the reaction of the 2-(4-methyl-2-phenylthiazole-5-carbonyl)-N-phenylhydrazinecarbothioamide with the appropriate hydrazonoyl chlorides. The structures of the newly synthesized compounds were established based on spectroscopic evidences and their alternative syntheses. Thirteen new 1,3,4-thiadiazoles have been evaluated for their anticancer activity against liver carcinoma cell line (HepG2). Also, their structure activity relationship (SAR) was studied. The 1,3,4-thiadiazoles 12d, $\mathbf{1 2 c}, \mathbf{6 g}, \mathbf{1 8 b}, \mathbf{6 c}$, and $\mathbf{6 f}\left(\mathrm{IC}_{50}=0.82,0.91,1.06,1.25,1.29\right.$ and $1.88 \mu \mathrm{M}$, respectively) have promising antitumor activity against liver carcinoma cell line (HepG2).
\end{abstract}

Keywords: thiazoles; thiadiazoles; hydrazonoyl chlorides; anticancer activity; structure activity relationship

\section{Introduction}

Cancer is a devastating and most common life-threatening disease representing a major health problem for many decades.The clinical application of chemotherapy still considered as a major compartment in treating cancer, however, is often limited by the severity of the side effects and the development of tumor cell resistance against these cytotoxic agents. Clinical administration of high doses of anticancer drugs to overcome resistance leads to severe toxicities [1]. Therefore, the development of novel effective anticancer drugs and strategies is eagerly being pursued.

Also, it was reported that Liver cancer is ranked in the top ten human cancers worldwide and among the top five of cancers in terms of mortality [2,3]. A literature survey revealed that a great deal of interest has been focused on the synthesis of functionalized thiazole derivatives due to their synthetic and biological potentialities as antihypertention [4], antifungal [5], antimicrobial [6,7], anti-inflammatory [8], antioxidant [9], antitubercular [10], and anticancer agents [11-14]. 1,3,4-Thiadiazole derivatives have attracted considerable interest due to their wide spectra of biological activities such as antibacterial, antifungal, antituberculosis, antihepatitis B viral, antileishmanial, anti-inflammatory, analgesic, CNS depressant, antioxidant, antidiabetic, molluscicidal, antihypertensive, diuretic, analgesic, antimicrobial, antitubercular, anticonvulsant and anticancer activities [15-24]. These important biological activities encouraged several research groups to find out different methods for synthesis of new thiadiazoles using different synthones, such as thiosemicarbazides, thiocarbazides, dithiocarbazates, thioacylhydrazines, acylhydrazines, 
and bithioureas [25]. In the light of the above-mentiond findings and in continuation of our efforts to synthesize new bioactive compounds [26-34], this work aims to synthesis a new series of thiazoles and 1,3,4-thiadiazoles bearing thiazole moiety and to study their anticancer activity against Liver carcinoma cell line (HepG2).

\section{Results and Discussion}

\subsection{Chemistry}

2-(4-Methyl-2-phenylthiazole-5-carbonyl)-N-phenylhydrazinecarbothioamide (3) was prepared as previously described, via reaction of 4-methyl-2-phenylthiazole-5-carbohydrazide (2) with phenyl isothiocyanate in EtOH as depicted in Scheme 1[35].

The presence of the thioamidehydrazine moiety as a side chain in compound 3 prompted us to utilize it for constructing 1,3,4-thiadiazole ring via its reaction with hydrazonoyl chlorides. Thus, reaction of compound 3 with the appropriate 2-oxo- $N$ '-arylpropanehydrazonoyl chlorides 4a-g [36] under reflux in ethanol in the presence of triethylamine as a basic catalyst led to formation of the respective 1,3,4-thiadiazoles 6a-g, rather than thiadiazines 7a-g or 1,3-thiazoles 8a-g (Scheme 1). The elemental analysis together with the data derived from IR, ${ }^{1} \mathrm{HNMR}$ and mass spectra are in agreement with the proposed structure $\mathbf{6}$. The IR spectra of products 6 showed in each case the presence of two absorption bands around $1700,1650 \mathrm{~cm}^{-1}$ for the two carbonyl groups, in addition to another band near $v 3350 \mathrm{~cm}^{-1}$ for the NH function. The ${ }^{1} \mathrm{HNMR}$ spectra of 6 revealed the presence of broad singlet signals assigned for the NH proton near $\delta 11.19 \mathrm{ppm}$, in addition to the expected signals for the protons of the $\mathrm{CH}_{3}$ group, the acetyl group at position-2 of the 1,3,4-thiadiazole ring and the aryl protons. The mass spectrum of each of products 6 revealed the presence of a molecular ion peak (see experimental section).<smiles></smiles>

1

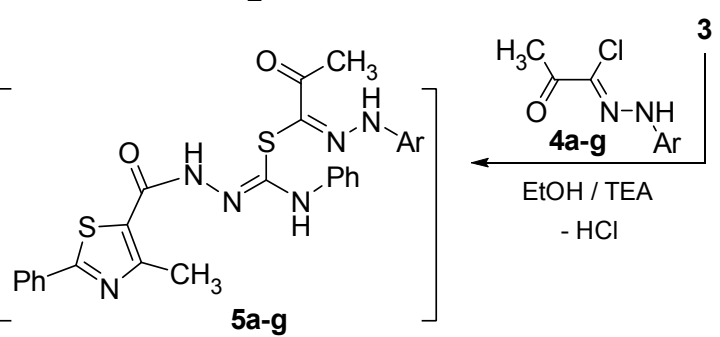
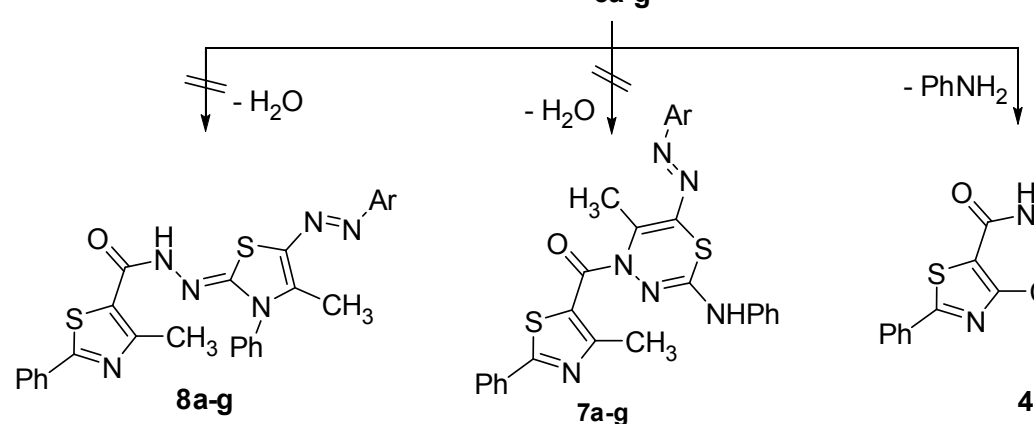<smiles></smiles><smiles></smiles>

\begin{tabular}{l|lllllll} 
4-8 & $\mathbf{a}$ & $\mathbf{b}$ & $\mathbf{c}$ & $\mathbf{d}$ & $\mathbf{e}$ & $\mathbf{f}$ & $\mathbf{g}$ \\
\hline $\mathrm{Ar}$ & $\mathrm{Ph}$ & $4-\mathrm{MePh}$ & 4-CIPh & 4-MeOPh & 3-CIPh & 4-BrPh & 2,4-(Cl) $\mathrm{Ph}$
\end{tabular}<smiles>Cc1nc(-c2ccccc2)sc1-c1n[nH]c(=S)o1</smiles>

Scheme 1. Synthesis of thiadiazoles 6a-g

1,3,4-Thiadiazole 6a-g was assumed to be formed through the intramolecluar cyclization of $\mathrm{NH}$ group in the hydrazone moiety with the imino group in the non-isolable intermediates 5a-g, 
followed by elimination of aniline molecule to give the respective thiadiazole derivatives $6 \mathbf{a}-\mathrm{g}$ (Scheme 1).

The structure of 6 was proved chemically via an alternative method (Scheme 1). Thus, the reaction of 5-(4-methyl-2-phenylthiazol-5-yl)-1,3,4-oxadiazole-2(3H)-thione(9)[35] with 4a in ethanol in the presence of triethylamine under reflux led to formation of product which is identical in all respects (mp, mixed $\mathrm{mp}$ and IR) with compound $\mathbf{6 a}$.

Next, in order to test of the biological activities of a vast array of these compounds, we reacted compound 3 with the appropriate hydrazonoyl chlorides 10a-d [36], under the same experimental conditions, which gave the corresponding 1,3,4-thiadiazole derivatives 12a-d(Scheme 2).

The IR, and ${ }^{1} \mathrm{H}-\mathrm{NMR}$ spectra of $\mathbf{1 2 a}$ taken as an example of the prepared series, revealed the presence of the ester group and the disappearance of the hydrazone-NH function. Also, the mass spectrum of the reaction products 12a-d showed, in each case, a peak corresponding to their molecular ions.

The structure assigned for product $\mathbf{1 2}$ was further evidenced via an alternative method. Thus, reaction of ethyl 4-methyl-2-phenylthiazole-5-carboxylate (1) with 1,3,4-thiadiazole 15 [37] in ethanol under reflux, afforded a product which is typical in all respects ( $\mathrm{mp}$, mixed $\mathrm{mp}$ and IR) with that obtained from the reaction of $\mathbf{3}$ with 10a (Scheme 2). To account for the formation of the product 12, it was suggested that the reaction of compound $\mathbf{3}$ with hydrazonoyl chloride $\mathbf{1 0}$ initially gave the intermediate 11, which underwent nucleophilic addition, followed by in situ cyclization via losing of one molecule of aniline (route a ) to give the final product 12. The other routes (b) and (c) outlined in Scheme 2 were excluded since they led to formation of products 13 and 14, which were completely different in all respects (IR, ${ }^{1} \mathrm{HNMR}$, mass spectra) from products $\mathbf{1 2}$.

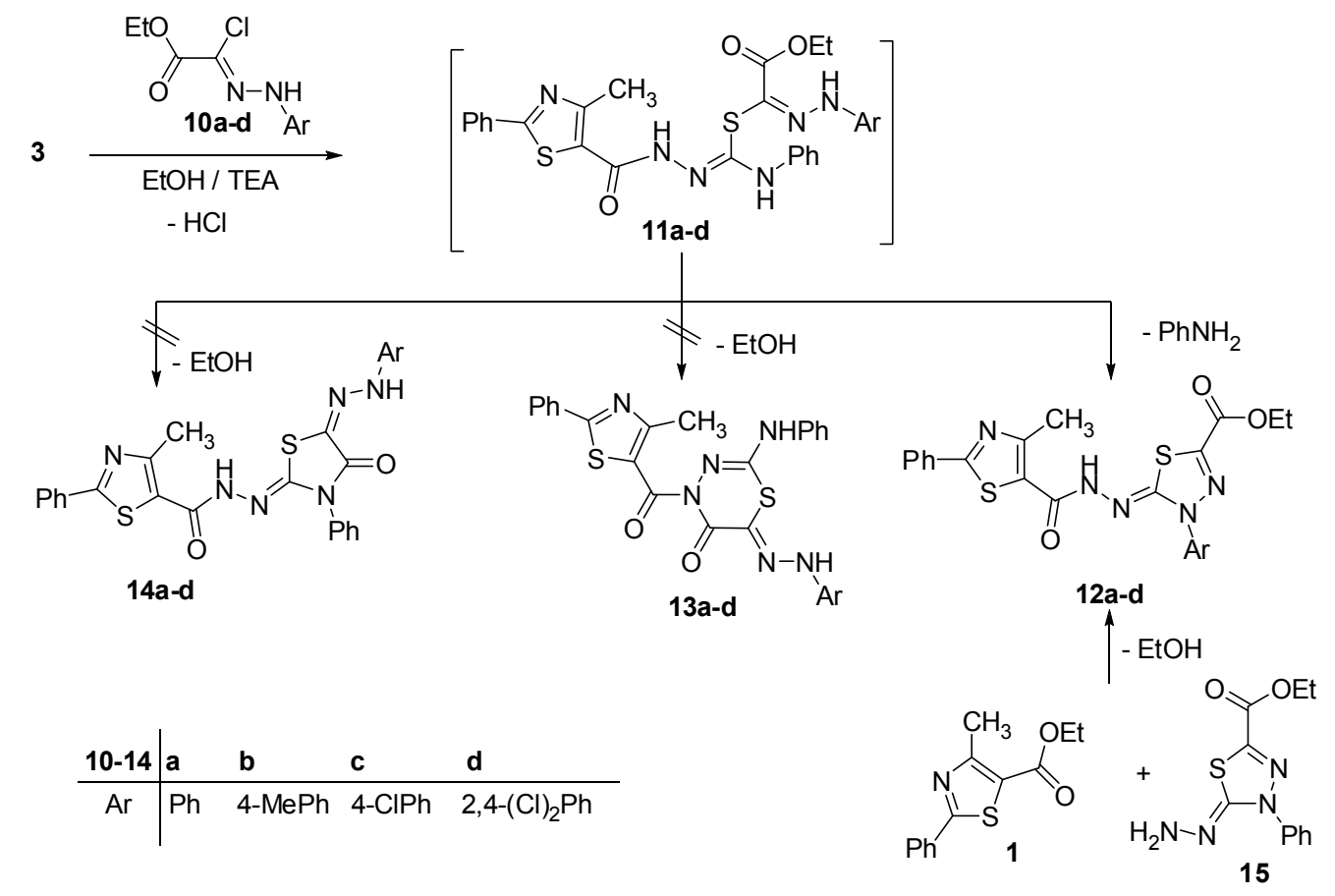

Scheme 2. Synthesis of thiadiazole derivatives 12a-d

Also, The reaction of compound $\mathbf{3}$ with hydrazonoyl halide of type $\mathbf{1 6}$ was studied. Thus refluxing compound 3 with the hydrazonoyl chloride $16 \mathbf{a}$ or $16 \mathbf{b}$ [36] under the same experimental conditions, afforded the corresponding 1,3,4-thiadiazole derivatives 18a,b (Scheme 3). The ${ }^{1} \mathrm{HNMR}$ spectrum of compound 18a, revealed two $\mathrm{D}_{2} \mathrm{O}$-exchangeable signals at $\delta 10.18$ and 11.72 corresponding to two $\mathrm{NH}$ protons, in addition to an aromatic multiplet in the region 7.02-7.78 ppm. Also, its mass spectrum of revealed a molecular ion peak at $\mathrm{m} / \mathrm{z}=512$ which is in complete agreement with the proposed structure (see Experimental). In addition, compound 18a was proved chemically via an alternative method from the reaction of compound 9 with 16a which gave a product identical in all respects ( $\mathrm{mp}$, mixed $\mathrm{mp}$ and IR) with compound 18a. 


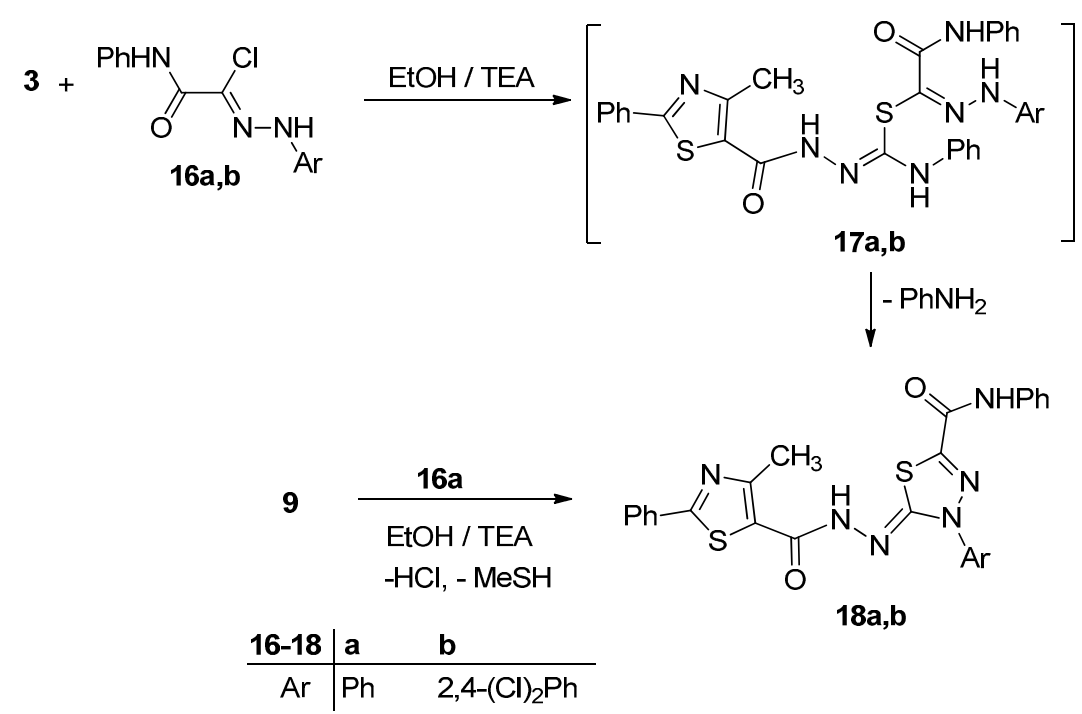

Scheme 3. Synthesis of thiadiazole derivatives $18 a, b$

The reaction of 4-methyl-2-phenylthiazole-5-carbohydrazide (2) with 2-oxo- $\mathrm{N}$-arylpropane hydrazonoyl chlorides $4 a-c$ in refluxing ethanol gave, in each case, the corresponding condensation product whose elemental analysis and spectra data(see Experimental) were consistent with structure 19 (Scheme 4). The IR spectra of the latter products exhibited a carbonyl and two NH absorption bands (see experimental part). Their ${ }^{1} \mathrm{HNMR}$ showed two $\mathrm{D}_{2} \mathrm{O}$ exchangeable signals of two $\mathrm{NH}$ groups in the regions $\delta 10.03-10.06$ and $\delta 10.57-10.59 \mathrm{ppm}$. Also, the mass spectra of the latter products confirmed the assigned structure 19 (Scheme 4 ).

Treatment of thioamide derivative 3 with the appropriate hydrazonoyl halides of type 19a-c in refluxing $\mathrm{EtOH}$ in the presence of TEA gave the corresponding thiadiazole derivatives 21a-c (Scheme 4). The structures of the isolated products 21a-c were elucidated on the basis of their spectral data and elemental analysis (see Experimental section). The latter products 21a-c were alternatively prepared by condensing 6a-c each with 4-methyl-2-phenylthiazole-5-carbohydrazide (2) in refluxing ethanol in quantitative yields (Scheme 4).

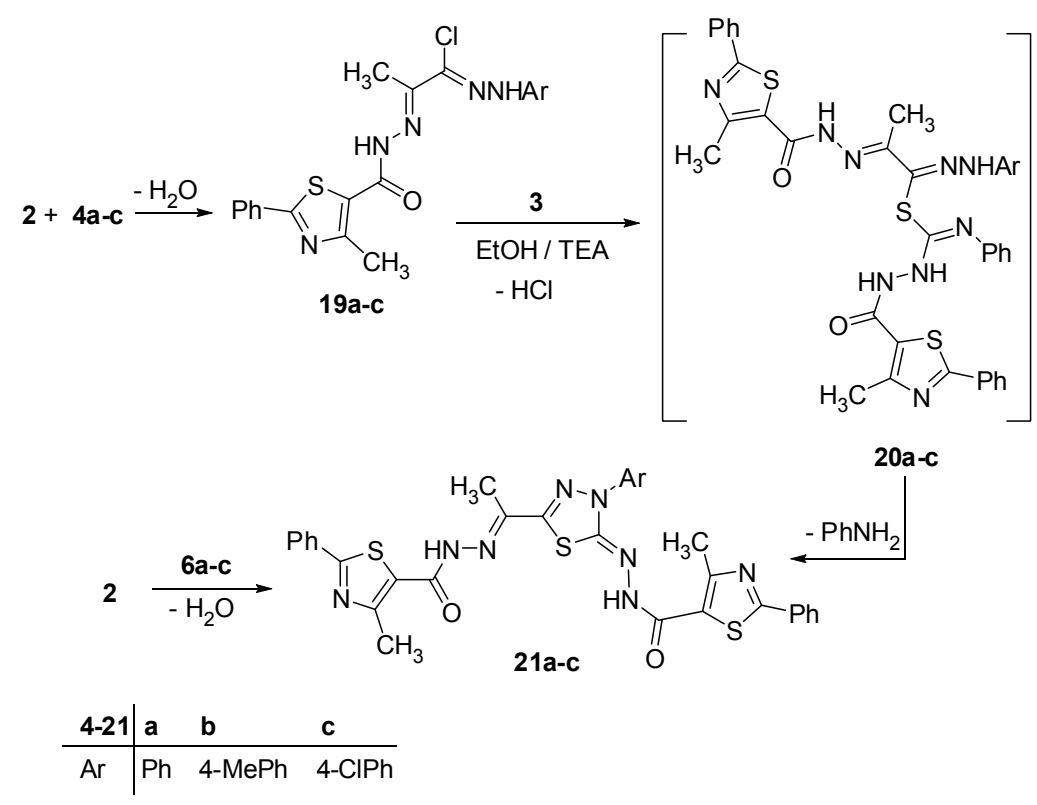

Scheme 4. Synthesis of thiadiazolederivatives 21a-c

Finally, the starting compound 3 was used for preparation of thiazole derivatives.

Thus, refluxing of compound 3 with 3-chloropentane-2,4-dione (22) or 2-chloro-3-oxo-Nphenylbutanamide (24) in $\mathrm{EtOH}$ in the presence of triethylamine afforded the thiazole derivatives 23 
and 25, respectively, as outlined in Scheme 5.The structure of compounds $\mathbf{2 3}$ and $\mathbf{2 5}$ were elucidated based on their elemental analysis and spectral data (see Experimental).

Also, thioamide derivative $\mathbf{3}$ reacted with phenacyl bromide $\mathbf{2 6}$ under the same experimental condition to afford one isolable product 27 named as $N^{\prime}$-(3,4-diphenylthiazol-2(3H)-ylidene)4-methyl-2-phenylthiazole-5-carbohydrazide (Scheme 5). The structure of the product 27 was established based on its elemental analysis and spectral data (see Experimental).

In a similar manner, thioamide derivative 3 reacted with ethyl chloroacetate (28) to afford a single product 29 that was identified as 4-methyl-N'-(4-oxo-3-phenylthiazolidin-2-ylidene)-2phenylthiazole-5-carbohydrazide (29) as outlined in Scheme 3. The structure of the isolated product 29 was established from its elemental analysis and spectral data. Its IR spectrum showed absorption bands at $v 3331(\mathrm{NH})$, and 1726, $1648(2 \mathrm{C}=\mathrm{O}) \mathrm{cm}^{-1}$, its ${ }^{1} \mathrm{H}-\mathrm{NMR}$ spectrum showed singlet signal at $\delta$ $4.23 \mathrm{ppm}$ due to the thiazolidinone $\left(\mathrm{CH}_{2}\right)$ group (see Experimental section).

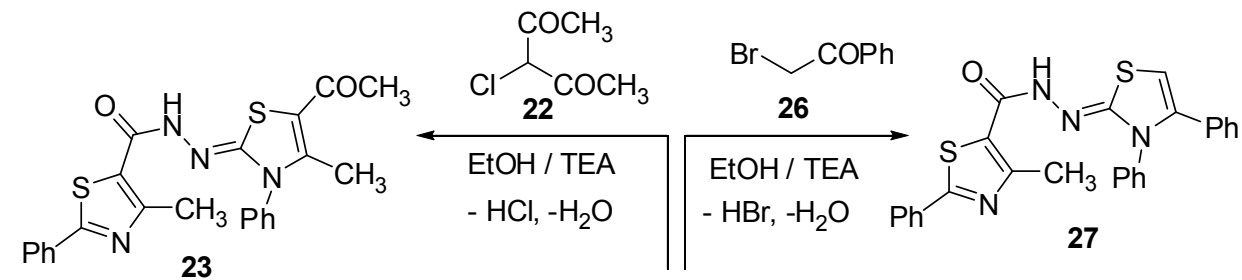<smiles></smiles>

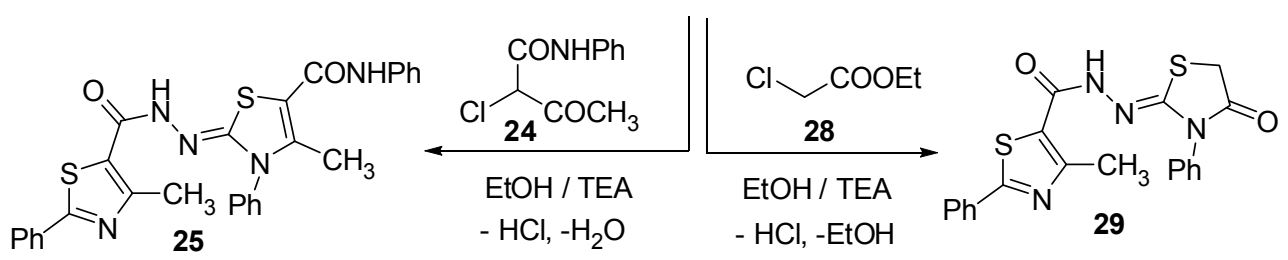

Scheme 5.Synthesis of thiazole derivatives 23, 25, 27and 29.

\subsection{Cytotoxic activity}

The Literature survey showed that many derivatives of thiazole and 1,3,4-thiadiazole have antitumor activity with excellent IG50 as depicted in Figure 1 [38-42].

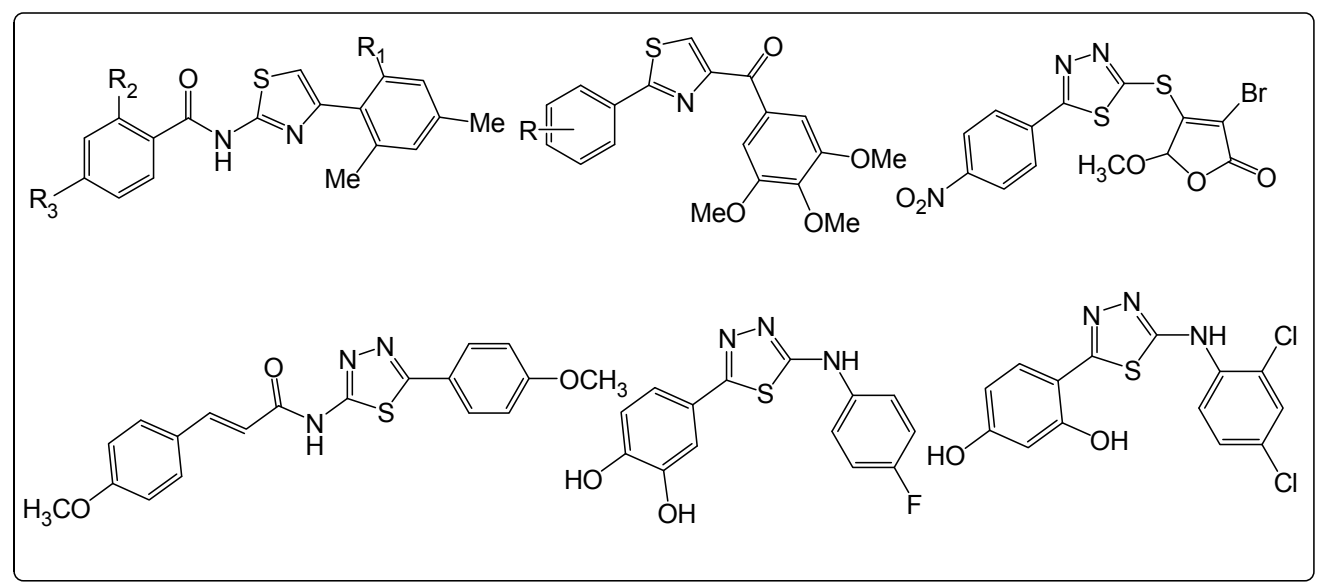

Figure 1. Lead compounds among thiazole and thiadiazole derivatives with anticancer activity In view of these facts, the antitumor activity of the synthesized compounds was determined against a liver carcinoma cell line HepG2. Doxorubicin was used as a reference standard and showed $\mathrm{IC}_{50}=0.72 \mu \mathrm{M}$ against a liver carcinoma cell line. Data generated were used to plot a dose-response 
curve of which the concentration $(\mu \mathrm{M})$ of test compounds required to kill $50 \%$ of cell population (IC50) was determined. Cytotoxic activity was expressed as the mean IC $_{50}$ of three independent experiments. The results depicted in table $\mathbf{1}$.

Table 1. Cytotoxic activities of tested compounds against liver carcinoma cell line (HepG2)

\begin{tabular}{|l|l|l|l|}
\hline Sample Number & $\mathbf{R}$ & $\mathbf{X}$ & $\mathbf{I C}_{\mathbf{5 0}}(\boldsymbol{\mu M})$ \\
\hline Doxorubicin & ---- & ---- & 0.72 \\
\hline $\mathbf{6 a}$ & $\mathrm{Ac}$ & $\mathrm{H}$ & 9.89 \\
\hline $\mathbf{6 b}$ & $\mathrm{Ac}$ & $4-\mathrm{Me}$ & 39.06 \\
\hline $\mathbf{6 c}$ & $\mathrm{Ac}$ & $4-\mathrm{Cl}$ & 1.29 \\
\hline $\mathbf{6 d}$ & $\mathrm{Ac}$ & $4-\mathrm{OMe}$ & 64.35 \\
\hline $\mathbf{6 e}$ & $\mathrm{Ac}$ & $3-\mathrm{Cl}$ & 4.03 \\
\hline $\mathbf{6 f}$ & $\mathrm{Ac}$ & $4-\mathrm{Br}$ & 1.88 \\
\hline $\mathbf{6 g}$ & $\mathrm{Ac}$ & $2,4-(\mathrm{Cl})_{2}$ & 1.06 \\
\hline $\mathbf{1 2 a}$ & $\mathrm{CO} 2 \mathrm{Et}$ & $\mathrm{H}$ & 4.70 \\
\hline $\mathbf{1 2 b}$ & $\mathrm{CO}_{2} \mathrm{Et}$ & $4-\mathrm{Me}$ & 32.46 \\
\hline $\mathbf{1 2 c}$ & $\mathrm{CO}_{2} \mathrm{Et}$ & $4-\mathrm{Cl}$ & 0.91 \\
\hline $\mathbf{1 2 d}$ & $\mathrm{CO}_{2} \mathrm{Et}$ & $2,4-(\mathrm{Cl})_{2}$ & 0.82 \\
\hline $\mathbf{1 8 a}$ & $\mathrm{CONHPh}$ & $\mathrm{H}$ & 6.79 \\
\hline $\mathbf{1 8 b}$ & $\mathrm{CONHPh}$ & $2,4-(\mathrm{Cl})_{2}$ & 1.25 \\
\hline
\end{tabular}

The results revealed that most of the tested compounds showed a great variable activity compared to reference drug as shown in Table 1 . The order of activity of the newly synthesized compounds was as follow: $6 \mathrm{~d}<\mathbf{6 b}<\mathbf{1 2 b}<\mathbf{6 a}<\mathbf{1 8 a}<\mathbf{1 2 a}<\mathbf{6 e}<\mathbf{6 f}<\mathbf{6 c}<\mathbf{1 8 b}<\mathbf{6 g}<\mathbf{1 2 c}<\mathbf{1 2 d}$ These results lead to the following conclusions.

- The thiadiazole derivatives $12 \mathrm{~d}, \mathbf{1 2 c}, \mathbf{6 g}, \mathbf{1 8 b}, \mathbf{6 c}$ and $\mathbf{6 f}$ showed high antitumor activity, and the thiadiazole derivatives $6 \mathbf{e}, \mathbf{1 2 a}, \mathbf{1 8 a}$ and $\mathbf{6 a}$ revealed moderate antitumor activity, while the thiadiazole derivatives $\mathbf{1 2 b}, \mathbf{6 b}$ and $\mathbf{6 d}$ exhibited poor antitumor activity.

- The ester group $(\mathrm{Co} 2 \mathrm{Et})$ at position 2 of the thiadiazole ring is necessary to have higher antitumor activity than the acetyl and the N-phenylcarboxamide (CONHPh) groups.

- The presence of chlorine or bromine group (electron-withdrawing groups) at the position 2 or 4 in the aryl moiety of the thiadiazole ring as in the compounds $12 d, 12 c, 6 g, 18 b, 6 c$ and 6f increased the cytotoxic activity. Also, halogen at positions 2 or 4 had more cytotoxic activity than halogen at position 3 .

- While presence of electron-donating groups such as methyl or methoxy at the position 4 as in the compounds $\mathbf{1 2 b}, \mathbf{6 b}$ and $\mathbf{6 d}$ decresed the cytotoxic activity.

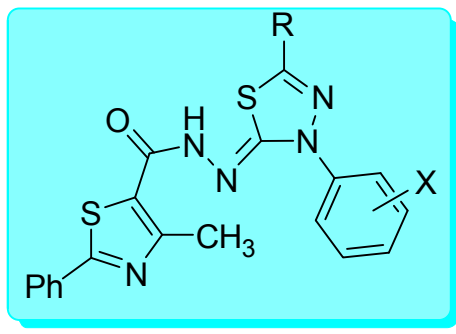

6a-g, 12a-d, and 18a,b

The influence of substituents on the observed biological activity:

a: Type of $X=$ electron withdrawing groups $>$ electron donating groups

b: Position of X: $4>3$

Figure 2. Structure-activity relationship of tested compounds against liver carcinoma cell line (HepG2) 


\section{Materials and Methods}

\subsection{Chemistry}

3.1.1. General

Melting points were measured on an Electrothermal IA 9000 series digital melting point apparatus. IR spectra were measured on PyeUnicam SP 3300 and Shimadzu FTIR 8101 PC infrared spectrophotometers in potassium bromide discs. NMR spectra were meaured on a Varian Mercury VX-300 NMR spectrometer operating at $300 \mathrm{MHz}\left({ }^{1} \mathrm{H}-\mathrm{NMR}\right)$ and run in deuterated dimethylsulfoxide (DMSO-d6). Chemical shifts were related to that of the solvent. Mass spectra were recorded on a Shimadzu GCMS-QP1000 EX mass spectrometer at $70 \mathrm{eV}$. Elemental analyses were measured by using a German made Elementarvario LIII CHNS analyzer. Antitumor activity of the products was measured at the Regional Center for Mycology and Biotechnology at Al-Azhar University, Cairo, Egypt. 2-(4-Methyl-2-phenylthiazole-5-carbonyl)-N-phenylhydrazine carbothioamide (3) [35], 5-(4-methyl-2-phenylthiazol-5-yl)-1,3,4-oxadiazole-2(3H)-thione (9)[35], hydrazonoyl halides 4a-g, 10a-d and 16a,b [36], and ethyl 5-hydrazono-4-phenyl-4,5-dihydro-1,3,4thiadiazole-2-carboxylate (15) [37] were prepared as reported in the respective literature.

\subsection{Synthetic Procedures}

3.2.1. Synthesis of 1,3,4-thiadiazole derivatives (6a-g, 12a-d and 18a,b).

General procedure. A mixture of compound $3(0.368 \mathrm{~g}, 1 \mathrm{mmol})$ and the appropriate hydrazonoyl chlorides $4 \mathbf{a}-\mathrm{g}$ or $10 \mathbf{a}-\mathbf{d}$ or $\mathbf{1 6} \mathbf{a}, \mathbf{b}(1 \mathrm{mmol})$ in ethanol $(20 \mathrm{~mL})$ containing triethylamine $(0.1 \mathrm{~g}, 1 \mathrm{mmol})$ was refluxed for $4-6 \mathrm{~h}$. (monitored by TLC). The formed solid product was filtered, washed with methanol, dried and recrystallized from the proper solvents to afford products $6 \mathbf{a}-\mathbf{g}$, 10a-d and 18a,b, respectively. The physical constants and spectral data of the obtained products are listed below:

3.2.1.1. $\quad N^{\prime}$-(5-Acetyl-3-phenyl-1,3,4-thiadiazol-2(3H)-ylidene)-4-methyl-2-phenylthiazole-5carbohydrazide (6a). Yellow solid (73\%); m.p. 163-165 C (EtOH); IR (KBr) v 3317 (NH), 3038,2951 $(\mathrm{CH}), 1701,1647(2 \mathrm{C}=\mathrm{O}), 1593(\mathrm{C}=\mathrm{N}) \mathrm{cm}^{-1} ;{ }^{1} \mathrm{H}-\mathrm{NMR}$ (DMSO-d6) $\delta 2.44\left(\mathrm{~s}, 3 \mathrm{H}, \mathrm{CH}_{3}\right), 2.74\left(\mathrm{~s}, 3 \mathrm{H}, \mathrm{CH}_{3}\right)$, 6.92-8.00 (m, 10H, ArH), 11.19 (s, br, 1H, D2O-exchangeable NH); MS m/z (\%) 435 (M+, 10), 381 (13), 274 (56), 118 (31), 92 (100), 65 (38). Anal. Calcd. for $\mathrm{C}_{21} \mathrm{H}_{17} \mathrm{~N}_{5} \mathrm{O}_{2} \mathrm{~S}_{2}$ (435.52): C, 57.91; H, 3.93; N, 16.08. Found C, 57.86; H, 3.84; N, 16.00\%.

3.2.1.2. $\quad N^{\prime}$-(5-Acetyl-3-(p-tolyl)-1,3,4-thiadiazol-2(3H)-ylidene)-4-methyl-2-phenylthiazole-5carbohydrazide (6b).Yellow solid (75\%); m.p. 149-151 ${ }^{\circ} \mathrm{C}(\mathrm{EtOH}) ; \mathrm{IR}(\mathrm{KBr}) v 3334(\mathrm{NH}), 3019,2920$ $(\mathrm{CH}), 1699,1648(2 \mathrm{C}=\mathrm{O}), 1597(\mathrm{C}=\mathrm{N}) \mathrm{cm}^{-1} ;{ }^{1} \mathrm{H}-\mathrm{NMR}$ (DMSO-d6) $\delta 2.31\left(\mathrm{~s}, 3 \mathrm{H}, \mathrm{CH}_{3}\right), 2.44\left(\mathrm{~s}, 3 \mathrm{H}, \mathrm{CH}_{3}\right)$, $2.73\left(\mathrm{~s}, 3 \mathrm{H}, \mathrm{CH}_{3}\right), 6.98-7.89$ (m, 9H, ArH), 11.18 (s, br, 1H, D2O-exchangeable NH); MS m/z (\%) 449 $\left(\mathrm{M}^{+}, 45\right), 372$ (54), 200 (27), 104 (36), 80 (100), 64 (35). Anal. Calcd. for $\mathrm{C}_{22} \mathrm{H}_{19} \mathrm{~N}_{5} \mathrm{O}_{2} \mathrm{~S}_{2}(449.55)$ : C, 58.78; $\mathrm{H}, 4.26 ; \mathrm{N}, 15.58$. Found C, 58.65; H, 4.17; N, $15.46 \%$.

3.2.1.3. $\quad N^{\prime}$-(5-Acetyl-3-(4-chlorophenyl)-1,3,4-thiadiazol-2(3H)-ylidene)-4-methyl-2-phenylthiazole-5-carbohydrazide (6c). Brown solid (75\%); m.p. 171-173 ${ }^{\circ} \mathrm{C}(\mathrm{EtOH})$; IR (KBr) $v 3325(\mathrm{NH})$, 3013, 2926 (CH), 1698, 1655 (2C=O), $1594(\mathrm{C}=\mathrm{N}) \mathrm{cm}^{-1},{ }^{1} \mathrm{H}-\mathrm{NMR}$ (DMSO-d6) $\delta 2.45$ (s, 3H, CH3), $2.76(\mathrm{~s}$, $\left.3 \mathrm{H}, \mathrm{CH}_{3}\right), 6.93-7.96$ (m, 9H, ArH), 11.25 (s, br, 1H, D $\mathrm{O}_{2}$-exchangeable $\left.\mathrm{NH}\right)$; MS m/z (\%) $471\left(\mathrm{M}^{+}+2,14\right)$, $469\left(\mathrm{M}^{+}, 45\right), 396$ (57), 200 (17), 80(100), 64 (89). Anal. Calcd. for $\mathrm{C}_{21} \mathrm{H}_{16} \mathrm{ClN}_{5} \mathrm{O}_{2} \mathrm{~S}_{2}$ (469.97): C, 53.67; H, 3.43; N, 14.90. Found C, 53.52; H, 3.37; N, 14.82\%.

3.2.1.4. $\quad N^{\prime}$-(5-Acetyl-3-(4-methoxyphenyl)-1,3,4-thiadiazol-2(3H)-ylidene)-4-methyl-2-phenylthiazole-5-carbohydrazide(6d). Brown solid (68\%); m.p. $143-145^{\circ} \mathrm{C}(\mathrm{EtOH}) ; \mathrm{IR}(\mathrm{KBr}) v 3328(\mathrm{NH})$, 3031, 2923 (CH), 1697, 1653 (2C=O), 1596 (C=N) cm ${ }^{-1} ;{ }^{1} \mathrm{H}-\mathrm{NMR}$ (DMSO-d6) $\delta 2.45$ (s, 3H, CH3), 2.75 (s, $\left.3 \mathrm{H}, \mathrm{CH}_{3}\right), 3.76\left(\mathrm{~s}, 3 \mathrm{H}, \mathrm{OCH}_{3}\right), 6.99-7.99(\mathrm{~m}, 9 \mathrm{H}, \mathrm{ArH}), 11.29$ (s, br, 1H, D2O-exchangeable $\left.\mathrm{NH}\right)$; $\mathrm{MS}$ $m / z(\%) 465\left(\mathrm{M}^{+}, 39\right), 334$ (87), 200 (63), 122 (80), 77 (100), 64 (45). Anal. Calcd. forC ${ }_{22} \mathrm{H}_{19} \mathrm{~N}_{5} \mathrm{O}_{3} \mathrm{~S}_{2}$ (465.55): C, 56.76; H, 4.11; N, 15.04. Found C, 56.63; H, 4.04; N, 14.95\%.

3.2.1.5. $\quad N^{\prime}$-(5-Acetyl-3-(3-chlorophenyl)-1,3,4-thiadiazol-2(3H)-ylidene)-4-methyl-2-phenyl thiazole-5-carbohydrazide(6e).Yellow solid (70\%); m.p. 166-168 ${ }^{\circ} \mathrm{C}(\mathrm{EtOH})$; IR (KBr) $v 3431(\mathrm{NH})$, 
3025, $2932(\mathrm{CH}), 1698,1659(2 \mathrm{C}=\mathrm{O}), 1593(\mathrm{C}=\mathrm{N}) \mathrm{cm}^{-1} ;{ }^{1} \mathrm{H}-\mathrm{NMR}$ (DMSO-d $) \delta 2.44\left(\mathrm{~s}, 3 \mathrm{H}, \mathrm{CH}_{3}\right), 2.66(\mathrm{~s}$, $\left.3 \mathrm{H}, \mathrm{CH}_{3}\right), 6.98-7.90(\mathrm{~m}, 9 \mathrm{H}, \mathrm{ArH}), 11.23\left(\mathrm{~s}, \mathrm{br}, 1 \mathrm{H}, \mathrm{D}_{2} \mathrm{O}-\right.$ exchangeable $\left.\mathrm{NH}\right)$; $\mathrm{MS} \mathrm{m} / z(\%) 471\left(\mathrm{M}^{+}+2,10\right)$, 469 (M+, 34), 334 (46), 200 (28), 132 (48), 80 (100), 64 (68). Anal. Calcd. for $\mathrm{C}_{21} \mathrm{H}_{16} \mathrm{ClN}_{5} \mathrm{O}_{2} \mathrm{~S}_{2}$ (469.97): C, $53.67 ; \mathrm{H}, 3.43 ; \mathrm{N}, 14.90$. Found C, 53.60; H, 3.36; N, 14.79\%.

3.2.1.6. $\quad N^{\prime}$-(5-Acetyl-3-(4-bromophenyl)-1,3,4-thiadiazol-2(3H)-ylidene)-4-methyl-2-phenyl thiazole-5-carbohydrazide (6f). Brown solid (73\%); m.p. 160-162 ${ }^{\circ} \mathrm{C}(\mathrm{EtOH})$; IR (KBr) $v 3429(\mathrm{NH})$, 3012, $2924(\mathrm{CH}), 1696,1654(2 \mathrm{C}=\mathrm{O}), 1594(\mathrm{C}=\mathrm{N}) \mathrm{cm}^{-1} ;{ }^{1} \mathrm{H}-\mathrm{NMR}$ (DMSO-d $) \delta 2.44\left(\mathrm{~s}, 3 \mathrm{H}, \mathrm{CH}_{3}\right), 2.65$ (s, $\left.3 \mathrm{H}, \mathrm{CH}_{3}\right)$, 6.95-7.94 (m, 9H, ArH), 11.25 (s, br, 1H, D $2 \mathrm{O}$-exchangeable NH); MS m/z (\%) $516\left(\mathrm{M}^{+}+2,51\right)$, $514\left(\mathrm{M}^{+}, 53\right), 325$ (76), 172 (44),91 (80), 80 (100), 64 (47). Anal. Calcd. for $\mathrm{C}_{21} \mathrm{H}_{16} \mathrm{BrN}_{5} \mathrm{O}_{2} \mathrm{~S}_{2}$ (514.42): C, 49.03; H, 3.14; N, 13.61. Found C, 48.93; H, 3.12; N, 13.53\%.

3.2.1.7. $\quad N^{\prime}$-(5-Acetyl-3-(2,4-dichlorophenyl)-1,3,4-thiadiazol-2(3H)-ylidene)-4-methyl-2-phenyl thiazole-5-carbohydrazide(6g). Brown solid (77\%); m.p. 181-183 ${ }^{\circ} \mathrm{C}$ (EtOH/dioxane); IR (KBr) $v 3318$ $(\mathrm{NH}), 3088,2926(\mathrm{CH}), 1699,1671(2 \mathrm{C}=\mathrm{O}), 1597(\mathrm{C}=\mathrm{N}) \mathrm{cm}^{-1} ;{ }^{1} \mathrm{H}-\mathrm{NMR}$ (DMSO- $\left.d_{6}\right) \delta 2.47\left(\mathrm{~s}, 3 \mathrm{H}, \mathrm{CH}_{3}\right)$, $2.67\left(\mathrm{~s}, 3 \mathrm{H}, \mathrm{CH}_{3}\right), 6.97-8.07$ (m, 8H, ArH), 11.19 (s, br, 1H, $\mathrm{D}_{2} \mathrm{O}$-exchangeable $\mathrm{NH}$ ); $\mathrm{MS} \mathrm{m} / \mathrm{z}(\%) 504$ $\left(\mathrm{M}^{+}, 14\right), 407$ (33), 161 (14), 80 (99), 64 (100). Anal. Calcd. for $\mathrm{C}_{21} \mathrm{H}_{15} \mathrm{Cl}_{2} \mathrm{~N}_{5} \mathrm{O}_{2} \mathrm{~S}_{2}$ (504.41): C, 50.00; H, 3.00; N, 13.88. Found C, 49.88; H, 2.92; N, 13.75\%.

3.2.1.8. Ethyl 5-(2-(4-methyl-2-phenylthiazole-5-carbonyl)hydrazono)-4-phenyl-4,5-dihydro-1,3,4thiadiazole-2-carboxylate (12a). Yellow solid (71\%); m.p. 137-139 ${ }^{\circ} \mathrm{C}(\mathrm{EtOH}) ; \mathrm{IR}(\mathrm{KBr}) v 3432(\mathrm{NH})$, 3035, $2923(\mathrm{CH}), 1749,1659(2 \mathrm{C}=\mathrm{O}), 1597(\mathrm{C}=\mathrm{N}) \mathrm{cm}^{-1} ;{ }^{1} \mathrm{H}-\mathrm{NMR}$ (DMSO- $\left.d_{6}\right) \delta 1.20(\mathrm{t}, 3 \mathrm{H}, J=7.1 \mathrm{~Hz}$, $\left.\mathrm{CH}_{2} \mathrm{CH}_{3}\right), 2.74\left(\mathrm{~s}, 3 \mathrm{H}, \mathrm{CH}_{3}\right), 4.21\left(\mathrm{q}, 2 \mathrm{H}, J=7.1 \mathrm{~Hz}, \mathrm{CH}_{2} \mathrm{CH}_{3}\right), 7.00-8.01(\mathrm{~m}, 10 \mathrm{H}, \mathrm{ArH}), 10.72(\mathrm{~s}, \mathrm{br}, 1 \mathrm{H}$, $\mathrm{D}_{2} \mathrm{O}$-exchangeable NH); MS m/z (\%): 465 (M+, 27), 334 (50), 200 (34), 104 (40), 80 (100), 64 (37). Anal. Calcd. for $\mathrm{C}_{22} \mathrm{H}_{19} \mathrm{~N}_{5} \mathrm{O}_{3} \mathrm{~S}_{2}$ (465.55): C, 56.76; H, 4.11; N, 15.04. Found C, 56.69; H, 4.03; N, 15.01\%.

3.2.1.9. Ethyl 5-(2-(4-methyl-2-phenylthiazole-5-carbonyl)hydrazono)-4-( $p$-tolyl)-4,5-dihydro-1,3,4thiadiazole-2-carboxylate (12b). Yellow solid (70\%); m.p. 147-149 ${ }^{\circ} \mathrm{C}(\mathrm{EtOH})$; IR (KBr) $v 3424(\mathrm{NH})$, 3058, $2925(\mathrm{CH}), 1749,1674(2 \mathrm{C}=\mathrm{O}), 1595(\mathrm{C}=\mathrm{N}) \mathrm{cm}^{-1} ;{ }^{1} \mathrm{H}-\mathrm{NMR}$ (DMSO-d $) \delta 1.20(\mathrm{t}, 3 \mathrm{H}, J=7.1 \mathrm{~Hz}$, $\left.\mathrm{CH}_{2} \mathrm{CH}_{3}\right), 2.26\left(\mathrm{~s}, 3 \mathrm{H}, \mathrm{CH}_{3}\right), 2.76\left(\mathrm{~s}, 3 \mathrm{H}, \mathrm{CH}_{3}\right), 4.19$ (q, $\left.2 \mathrm{H}, J=7.1 \mathrm{~Hz}, \mathrm{CH}_{2} \mathrm{CH}_{3}\right), 7.00-8.02(\mathrm{~m}, 9 \mathrm{H}, \mathrm{ArH})$, 10.73 (s, br, 1H, $\mathrm{D}_{2} \mathrm{O}$-exchangeable NH); MS m/z (\%) 479 (M+, 20), 367 (25), 251 (18), 80 (85), 64 (100). Anal. Calcd. for $\mathrm{C}_{23} \mathrm{H}_{21} \mathrm{~N}_{5} \mathrm{O}_{3} \mathrm{~S}_{2}$ (479.57): C, 57.60; H, 4.41; N, 14.60. Found C, 57.49; H, 4.33; N, 14.51\%. 3.2.1.10. Ethyl 4-(4-chlorophenyl)-5-(2-(4-methyl-2-phenylthiazole-5-carbonyl)hydrazono)-4,5dihydro-1,3,4-thiadiazole-2-carboxylate(12c). Yellow solid (73\%); m.p. 167-169 ${ }^{\circ} \mathrm{C}$ (EtOH/dioxane); IR (KBr) $v 3340(\mathrm{NH}), 3050,2927(\mathrm{CH}), 1748,1670(2 \mathrm{C}=\mathrm{O}), 1599(\mathrm{C}=\mathrm{N}) \mathrm{cm}^{-1} ;{ }^{1} \mathrm{H}-\mathrm{NMR}\left(\mathrm{DMSO}-d_{6}\right) \delta$ $1.23\left(\mathrm{t}, 3 \mathrm{H}, J=7.1 \mathrm{~Hz}, \mathrm{CH}_{2} \underline{\mathrm{C}}_{3}\right), 2.75\left(\mathrm{~s}, 3 \mathrm{H}, \mathrm{CH}_{3}\right), 4.22\left(\mathrm{q}, 2 \mathrm{H}, J=7.1 \mathrm{~Hz}, \mathrm{CH}_{2} \mathrm{CH}_{3}\right), 7.02-7.96(\mathrm{~m}, 9 \mathrm{H}$, $\mathrm{ArH}), 10.77$ (s, br, 1H, D2O-exchangeable NH); MS m/z (\%) $501\left(\mathrm{M}^{+}+2,13\right), 499\left(\mathrm{M}^{+}, 45\right), 363$ (39), 334 (100), 200 (35), 104 (30), 77 (50). Anal. Calcd. for $\mathrm{C}_{22} \mathrm{H}_{18} \mathrm{ClN}_{5} \mathrm{O}_{3} \mathrm{~S}_{2}$ (499.99): C, 52.85; H, 3.63; N, 14.01. Found C, 52.79; H, 3.60; N, 13.87\%.

3.2.1.11. Ethyl 4-(2,4-dichlorophenyl)-5-(2-(4-methyl-2-phenylthiazole-5-carbonyl)hydrazono)-4,5dihydro-1,3,4-thiadiazole-2-carboxylate (12d). Brown solid (75\%); m.p. 173-175 ${ }^{\circ} \mathrm{C}$ (EtOH/dioxane); IR (KBr) $v 3221(\mathrm{NH}), 3079,2926(\mathrm{CH}), 1749,1671(2 \mathrm{C}=\mathrm{O}), 1599(\mathrm{C}=\mathrm{N}) \mathrm{cm}^{-1} ;{ }^{1} \mathrm{H}-\mathrm{NMR}$ (DMSO-d $\left.d_{6}\right) \delta$ $1.24\left(\mathrm{t}, 3 \mathrm{H}, J=7.1 \mathrm{~Hz}, \mathrm{CH}_{2} \mathrm{CH}_{3}\right), 2.77\left(\mathrm{~s}, 3 \mathrm{H}, \mathrm{CH}_{3}\right), 4.23\left(\mathrm{q}, 2 \mathrm{H}, J=7.1 \mathrm{~Hz}, \mathrm{CH}_{2} \mathrm{CH}_{3}\right), 7.08-8.13(\mathrm{~m}, 8 \mathrm{H}$, $\mathrm{ArH}), 10.77$ (s, br, 1H, $\mathrm{D}_{2} \mathrm{O}-$ exchangeable NH); MS m/z (\%) $534\left(\mathrm{M}^{+}, 19\right), 449$ (78), 223(100), 200 (54), 104 (58), 80 (85). Anal. Calcd. for $\mathrm{C}_{22} \mathrm{H}_{17} \mathrm{Cl}_{2} \mathrm{~N}_{5} \mathrm{O}_{3} \mathrm{~S}_{2}$ (534.44): C, 49.44; H, 3.21; N, 13.10. Found C, 49.29; $\mathrm{H}, 3.16 ; \mathrm{N}, 13.02 \%$.

3.2.1.12. 5-(2-(4-Methyl-2-phenylthiazole-5-carbonyl)hydrazono)-N,4-diphenyl-4,5-dihydro-1,3,4thiadiazole-2-carboxamide (18a). Brown solid (76\%); m.p. 176-178 ${ }^{\circ} \mathrm{C}(\mathrm{EtOH} /$ dioxane); $\mathrm{IR}(\mathrm{KBr}) v$ 3427, $3343(2 \mathrm{NH}), 1672,1653(2 \mathrm{C}=\mathrm{O}), 1597(\mathrm{C}=\mathrm{N}) \mathrm{cm}^{-1}$; ${ }^{1} \mathrm{H}-\mathrm{NMR}$ (DMSO-d6) $\delta 2.75\left(\mathrm{~s}, 3 \mathrm{H}, \mathrm{CH}_{3}\right)$, 7.02-7.78 (m, 15H, ArH), 10.18(s, br, 1H, $\mathrm{D}_{2} \mathrm{O}$-exchangeable NH), 11.72 (s, br, 1H, $\mathrm{D}_{2} \mathrm{O}$-exchangeable $\mathrm{NH})$; MS m/z (\%) $512\left(\mathrm{M}^{+}, 8\right), 401$ (00), 282 (10), 150 (22), 92 (26), 65 (29). Anal. Calcd. For $\mathrm{C}_{26} \mathrm{H}_{20} \mathrm{~N}_{6} \mathrm{O}_{2} \mathrm{~S}_{2}$ (512.61): C, 60.92; H, 3.93; N, 16.39. Found C, 60.78; H, 3.85; N, 16.32\%.

3.2.1.13. 4-(2,4-Dichlorophenyl)-5-(2-(4-methyl-2-phenylthiazole-5-carbonyl)hydrazono)- $\mathrm{N}$ phenyl-4,5-dihydro-1,3,4-thiadiazole-2-carboxamide (18b). Brown solid (77\%); m.p. 186-188 $\mathrm{C}$ (Dioxane); IR (KBr) $v$ 3429, 3337(2NH), 1692, $1656(2 \mathrm{C}=\mathrm{O}), 1591(\mathrm{C}=\mathrm{N}) \mathrm{cm}^{-1} ;{ }^{1} \mathrm{H}-\mathrm{NMR}\left(\mathrm{DMSO}-d_{6}\right) \delta$ $2.76\left(\mathrm{~s}, 3 \mathrm{H}, \mathrm{CH}_{3}\right), 7.13-7.83(\mathrm{~m}, 13 \mathrm{H}, \mathrm{ArH}), 10.19\left(\mathrm{~s}, \mathrm{br}, 1 \mathrm{H}, \mathrm{D}_{2} \mathrm{O}-\right.$ exchangeable $\left.\mathrm{NH}\right), 11.77(\mathrm{~s}, \mathrm{br}, 1 \mathrm{H}$, 
$\mathrm{D}_{2} \mathrm{O}$-exchangeable NH); MS m/z (\%) 581 (M+, 38), 473 (64), 334 (72), 200 (35), 119 (65), 64 (100). Anal. Calcd. for $\mathrm{C}_{26} \mathrm{H}_{18} \mathrm{Cl}_{2} \mathrm{~N}_{6} \mathrm{O}_{2} \mathrm{~S}_{2}$ (581.50): C, 53.70; $\mathrm{H}, 3.12 ; \mathrm{N}, 14.45$. Found $\mathrm{C}, 53.62 ; \mathrm{H}, 3.03 ; \mathrm{N}, 14.32 \%$.

\subsubsection{Alternate synthesis of thiadiazole derivatives $6 a$ and $18 a$}

To a mixture of 5-(4-methyl-2-phenylthiazol-5-yl)-1,3,4-oxadiazole-2(3H)-thione (9) (0.275 g, 1 mmol) and hydrazonoyl chloride $4 \mathbf{a}$ or 16a $(1 \mathrm{mmol})$ in absolute EtOH $(25 \mathrm{~mL})$, was added triethylamine $(0.1 \mathrm{~g}, 0.14 \mathrm{~mL}, 1 \mathrm{mmol})$. The reaction mixture was stirred at room temperature till methyl mercaptan ceased to evolve (3h). The solvent was evaporated and the residue was treated with ice/ $\mathrm{HCl}$ mixture. The solid product was collected by filteration, washed with EtOH, dried, and recrystallized to give the respective compounds $\mathbf{6 a}$, and 18a, that was identical in all respects (m.p., mixed m.p. and IR spectra) with that obtained from reaction of $4 \mathbf{a}$ or $16 a$ with 3.

\subsubsection{Alternate synthesis of 12a}

A mixture of ethyl 4-methyl-2-phenylthiazole-5-carboxylate $(\mathbf{1})(0.247 \mathrm{~g}, 1 \mathrm{mmol})$ and ethyl 5-hydrazono-4-phenyl-4,5-dihydro-1,3,4-thiadiazole-2-carboxylate (15) $(0.264 \mathrm{~g}, 1 \mathrm{mmol})$ was refluxed in ethanol for $4 \mathrm{~h}$. The solid product that separated was filtered off, washed with water and finally recrystallized to give the corresponding product, 12a which was identical in all aspects (m.p., mixed m.p. and IR spectra) with those obtained from reaction of $\mathbf{3}$ with 10a.

\subsubsection{Synthesis of hydrazonoyl chlorides 19a-c}

A mixture of 4-methyl-2-phenylthiazole-5-carbohydrazide (2) $(2.33 \mathrm{~g}, 10 \mathrm{mmol})$ and the appropriate hydrazonoyl chlorides $4 a-c(10 \mathrm{mmol})$ in ethanol $(30 \mathrm{~mL})$ was refluxed for $3 \mathrm{hr}$. The resulting solid product was collected and recrystallized from the proper solvent to give the corresponding products19a-c.

3.2.4.1. 2-(2-(4-Methyl-2-phenylthiazole-5-carbonyl)hydrazono)- $N$ '-phenylpropanehydrazonoyl chloride (19a). Yellow solid (84\%); m.p. 188-190 ${ }^{\circ} \mathrm{C}(\mathrm{EtOH}) ; \mathrm{IR}(\mathrm{KBr}) v 3440,3316(2 \mathrm{NH}), 3036$, 2922(CH), $1640(\mathrm{C}=\mathrm{O}), 1599(\mathrm{C}=\mathrm{N}) \mathrm{cm}^{-1} ;{ }^{1} \mathrm{H}-\mathrm{NMR}$ (DMSO-d $) \delta 2.36\left(\mathrm{~s}, 3 \mathrm{H}, \mathrm{CH}_{3}\right), 2.76\left(\mathrm{~s}, 3 \mathrm{H}, \mathrm{CH}_{3}\right)$, 7.06-7.86 (m, 10H, ArH), 10.03 (s, br, 1H, $\mathrm{D}_{2} \mathrm{O}$-exchangeable NH), 10.57 (s, br, 1H, $\mathrm{D}_{2} \mathrm{O}$-exchangeable $\mathrm{NH}) ; \mathrm{MS} m / z(\%): 413\left(\mathrm{M}^{+}+2,12\right), 411\left(\mathrm{M}^{+}, 40\right), 375$ (48), 202 (100), 174 (45), 71 (26). Anal. calcd for $\mathrm{C}_{20} \mathrm{H}_{18} \mathrm{ClN} 5 \mathrm{OS}$ (411.91): C, 58.32; H, 4.40; N, 17.00.Found: C, 58.19; H, 4.37; N, 16.88\%.

3.2.4.2.

2-(2-(4-Methyl-2-phenylthiazole-5-carbonyl)hydrazono)- $N$ '-( $p$-tolyl)propanehydrazonoylchloride (19b). Yellow solid (86\%); m.p. 172-174 ${ }^{\circ} \mathrm{C}(\mathrm{EtOH}) ; \mathrm{IR}(\mathrm{KBr}) v$ 3437, $3313(2 \mathrm{NH})$, 3041, $2917(\mathrm{CH}), 1679(\mathrm{C}=\mathrm{O}), 1598(\mathrm{C}=\mathrm{N}) \mathrm{cm}^{-1}$; ${ }^{1} \mathrm{H}-\mathrm{NMR}$ (DMSO-d6) $\delta 2.24\left(\mathrm{~s}, 3 \mathrm{H}, \mathrm{CH}_{3}\right), 2.34(\mathrm{~s}, 3 \mathrm{H}$, $\left.\mathrm{CH}_{3}\right), 2.77$ (s, 3H, $\left.\mathrm{CH}_{3}\right), 7.08-7.99(\mathrm{~m}, 9 \mathrm{H}, \mathrm{ArH}), 10.06\left(\mathrm{~s}, \mathrm{br}, 1 \mathrm{H}, \mathrm{D}_{2} \mathrm{O}\right.$-exchangeable $\left.\mathrm{NH}\right), 10.59$ (s, br, 1H, $\mathrm{D}_{2} \mathrm{O}$-exchangeable NH); MS m/z (\%) $427\left(\mathrm{M}^{+}+2,10\right), 425\left(\mathrm{M}^{+}, 33\right), 389$ (26), 202 (81), 106 (100), 64 (66). Anal. calcd for $\mathrm{C}_{21} \mathrm{H}_{20} \mathrm{ClN} 5 \mathrm{OS}$ (425.93): C, 59.22; H, 4.73; N, 16.44. Found: C, 59.18; H, 4.65; N, $16.37 \%$.

3.2.4.3. $\quad N^{\prime}$-(4-Chlorophenyl)-2-(2-(4-methyl-2-phenylthiazole-5-carbonyl)hydrazono)propanehydrazonoyl chloride (19c).Yellow solid (87\%); m.p. 194-196 ${ }^{\circ} \mathrm{C}(\mathrm{DMF}) ; \mathrm{IR}(\mathrm{KBr}) v 3434,3319$ (2NH), 3044, 2926(CH), $1682(\mathrm{C}=\mathrm{O}), 1593(\mathrm{C}=\mathrm{N}) \mathrm{cm}^{-1}$; ${ }^{1} \mathrm{H}-\mathrm{NMR}$ (DMSO-d $) \delta 2.37\left(\mathrm{~s}, 3 \mathrm{H}, \mathrm{CH}_{3}\right), 2.77(\mathrm{~s}, 3 \mathrm{H}$, $\left.\mathrm{CH}_{3}\right), \quad 7.08-7.99(\mathrm{~m}, 9 \mathrm{H}, \mathrm{Ar}-\mathrm{H}), 10.06\left(\mathrm{~s}, \mathrm{br}, 1 \mathrm{H}, \mathrm{D}_{2} \mathrm{O}-\right.$-exchangeable $\left.\mathrm{NH}\right), 10.57(\mathrm{~s}, \mathrm{br}, 1 \mathrm{H}$, $\mathrm{D}_{2} \mathrm{O}$-exchangeable NH); MS m/z (\%) $446\left(\mathrm{M}^{+}, 8\right), 283$ (14), 202 (39), 104 (46), 80 (100), 64 (90). Anal. calcd for $\mathrm{C}_{20} \mathrm{H}_{17} \mathrm{Cl}_{2} \mathrm{~N}_{5} \mathrm{OS}$ (446.35): C, 53.82; H, 3.84; N, 15.69. Found: $\mathrm{C}, 53.75 ; \mathrm{H}, 3.79 ; \mathrm{N}, 15.58 \%$.

\subsubsection{Synthesis of 1,3,4-thiadiazole derivatives 21a-c.}

Method A: A mixture of compound $3(0.368 \mathrm{~g}, 1 \mathrm{mmol})$ and the appropriate hydrazonoyl chlorides 19a-c $(1 \mathrm{mmol})$ in ethanol $(20 \mathrm{~mL})$ containing triethylamine $(0.1 \mathrm{~g}, 1 \mathrm{mmol})$ was refluxed for $6 \mathrm{~h} .$. The formed solid produt was filtered, washed with methanol, dried and recrystallized from the suitable solvents to give corresponding products 21a-c.

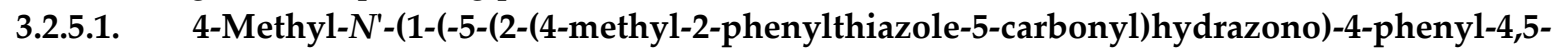
dihydro-1,3,4-thiadiazol-2-yl)ethylidene)-2-phenylthiazole-5-carbohydrazide (21a). Yellow solid 
(74\%); m.p. 162-164 C (EtOH); IR (KBr) v 3421, 3307 (2NH), 3031, $2951(\mathrm{CH}), 1649(\mathrm{C}=\mathrm{O}), 1596(\mathrm{C}=\mathrm{N})$ $\mathrm{cm}^{-1} ;{ }^{1} \mathrm{H}-\mathrm{NMR}\left(\mathrm{DMSO}-d_{6}\right) \delta 2.34\left(\mathrm{~s}, 3 \mathrm{H}, \mathrm{CH}_{3}\right), 2.66\left(\mathrm{~s}, 3 \mathrm{H}, \mathrm{CH}_{3}\right), 2.76\left(\mathrm{~s}, 3 \mathrm{H}, \mathrm{CH}_{3}\right), 6.97-8.14(\mathrm{~m}, 15 \mathrm{H}$, $\mathrm{ArH}), 10.18$ (s, br, 1H, D2O-exchangeable NH), 11.17 (s, br, 1H, D2O-exchangeable NH); MS m/z (\%) $650\left(\mathrm{M}^{+}, 34\right), 526$ (30), 416 (60), 358 (28), 104 (55), 64 (100). Anal. Calcd for $\mathrm{C}_{32} \mathrm{H}_{26} \mathrm{~N}_{8} \mathrm{O}_{2} \mathrm{~S}_{3}$ (650.80): C, 59.06; H, 4.03; N, 17.22. Found C, 58.94; H, 4.01; N, 17.07\%.

3.2.5.2. 4-Methyl-N'-(1-(5-(2-(4-methyl-2-phenylthiazole-5-carbonyl)hydrazono)-4-( $p$-tolyl)-4,5dihydro-1,3,4-thiadiazol-2-yl)ethylidene)-2-phenylthiazole-5-carbohydrazide(21b). Yellow solid (72\%); m.p. 149-151 ${ }^{\circ} \mathrm{C}(\mathrm{EtOH})$; IR (KBr) v 3422, $3328(2 \mathrm{NH}), 3053,2929(\mathrm{CH}), 1647$ (C=O), $1597(\mathrm{C}=\mathrm{N})$ $\mathrm{cm}^{-1} ;{ }^{1} \mathrm{H}-\mathrm{NMR}\left(\mathrm{DMSO}-d_{6}\right) \delta 2.26\left(\mathrm{~s}, 3 \mathrm{H}, \mathrm{CH}_{3}\right), 2.35(\mathrm{~s}, 3 \mathrm{H}, \mathrm{CH}), 2.65\left(\mathrm{~s}, 3 \mathrm{H}, \mathrm{CH}_{3}\right), 2.76\left(\mathrm{~s}, 3 \mathrm{H}, \mathrm{CH}_{3}\right)$, 6.91-8.03 (m, 14H, ArH), 10.18 (s, br, 1H, D2O-exchangeable NH), 11.14 (s, br, 1H, $\mathrm{D}_{2} \mathrm{O}$-exchangeable $\mathrm{NH})$; MS m/z (\%) $664\left(\mathrm{M}^{+}, 35\right), 553$ (60), 334 (19), 202 (65), 104 (85), 64 (100). Anal. Calcd for $\mathrm{C}_{33} \mathrm{H}_{28} \mathrm{~N}_{8} \mathrm{O}_{2} \mathrm{~S}_{3}$ (664.82): C, 59.62; H, 4.25; N, 16.85. Found C, 59.47; H, 4.17; N, 16.79\%.

3.2.5.3. $\quad N^{\prime}$-(3-(4-Chlorophenyl)-5-(1-(2-(4-methyl-2-phenylthiazole-5-carbonyl)hydrazono)-ethyl)1,3,4-thiadiazol-2(3H)-ylidene)-4-methyl-2-phenylthiazole-5-carbohydrazide (21c). Yellow solid (76\%); m.p. 191-193 C (Dioxane); IR (KBr) v 3424, 3312 (2NH), 3047, 2932 (CH), 1649 (C=O), 1599 $(\mathrm{C}=\mathrm{N}) \mathrm{cm}^{-1} ;{ }^{1} \mathrm{H}-\mathrm{NMR}\left(\mathrm{DMSO}-d_{6}\right) \delta 2.33\left(\mathrm{~s}, 3 \mathrm{H}, \mathrm{CH}_{3}\right), 2.66\left(\mathrm{~s}, 3 \mathrm{H}, \mathrm{CH}_{3}\right), 2.77\left(\mathrm{~s}, 3 \mathrm{H}, \mathrm{CH}_{3}\right), 6.90-8.11(\mathrm{~m}$, $14 \mathrm{H}, \mathrm{ArH}), 10.13$ (s, br, 1H, D2O-exchangeable NH), 11.19 (s, br, 1H, D $2 \mathrm{O}$-exchangeable $\mathrm{NH}$ ); MS m/z (\%) $686\left(\mathrm{M}^{+}+2,8\right), 684\left(\mathrm{M}^{+}, 26\right), 513$ (53), 368 (39), 257 (17), 104 (25), 64 (100). Anal. Calcd for $\mathrm{C}_{32} \mathrm{H}_{25} \mathrm{ClN}_{8} \mathrm{O}_{2} \mathrm{~S}_{3}(685.24): \mathrm{C}, 56.09 ; \mathrm{H}, 3.68 ; \mathrm{N}, 16.35$. Found C, 56.02; H, 3.58; N, $16.22 \%$.

3.2.6. Method B: A mixture of 4-methyl-2-phenylthiazole-5-carbohydrazide (2) (0.233 g, $1 \mathrm{mmol})$ and the appropriate 1,3,4-thiadiazoles $6 \mathrm{a}-\mathrm{c}(1 \mathrm{mmol})$ in ethanol $(10 \mathrm{~mL})$ was refluxed for $4 \mathrm{~h}$, allowed to cool and the solid product that formed was filtered off, washed with EtOH, dried and recrystallized from the proper solvent to give the corresponding product, 21a-c which were identical in all aspects (m.p., mixed m.p. and IR spectra) with those obtained from reaction of 3 with 19a-c.

\subsubsection{General procedure for the synthesis of thiazole derivatives $23,25,27$, and 29}

A mixture of compound $3(0.368 \mathrm{~g}, 1 \mathrm{mmol})$ and the appropriate $\alpha$-halo-compounds namely, 3-chloropentane-2,4-dione (22), 2-chloro-3-oxo-N-phenylbutanamide (24), 2-bromo-1-phenyl ethanone (26) and ethyl 2-chloroacetate (28) (1 mmol for each) in ethanol (20 $\mathrm{mL})$ containing triethylamine $(0.1 \mathrm{~g}, 1 \mathrm{mmol})$ was refluxed for 4-6 h. (monitored by TLC The solid product was filtered, washed with water, dried and recrystallized from the proper solvent to give the corresponding thiazole derivatives $23,25,27$ and 29 , respectively.

3.2.7.1. $\quad N^{\prime}$-(5-Acetyl-4-methyl-3-phenylthiazol-2(3H)-ylidene)-4-methyl-2-phenylthiazole-5carbohydrazide (23). Yellow solid (78\%); m.p. 155-157 C (EtOH); IR (KBr) v 3432 (NH), 3036, 2993 $(\mathrm{CH}), 1695,1648(2 \mathrm{C}=\mathrm{O}), 1590(\mathrm{C}=\mathrm{N}) \mathrm{cm}^{-1} ;{ }^{1} \mathrm{H}-\mathrm{NMR}\left(\mathrm{DMSO}-d_{6}\right) \delta 2.32\left(\mathrm{~s}, 3 \mathrm{H}, \mathrm{CH}_{3}\right), 2.46\left(\mathrm{~s}, 3 \mathrm{H}, \mathrm{CH}_{3}\right)$, $2.77\left(\mathrm{~s}, 3 \mathrm{H}, \mathrm{CH}_{3}\right), 6.91-7.86(\mathrm{~m}, 10 \mathrm{H}, \mathrm{ArH}), 10.61$ (s, br, 1H, D2O-exchangeable NH); MS m/z (\%) 448 $\left(\mathrm{M}^{+}, 57\right), 246$ (60), 176 (35), 104 (80), 77 (100). Anal. Calcd for $\mathrm{C}_{23} \mathrm{H}_{20} \mathrm{~N}_{4} \mathrm{O}_{2} \mathrm{~S}_{2}$ (448.56): C, 61.59; H, 4.49; $\mathrm{N}, 12.49$. Found C, 61.48; H, 4.36; N, 12.37\%.

3.2.7.2.4-Methyl-2-(2-(4-methyl-2-phenylthiazole-5-carbonyl)hydrazono)- $N$-3-diphenyl-2,3-dihydr othiazole-5-carboxamide (25). Yellow solid (79\%); m.p. 182-84 ${ }^{\circ} \mathrm{C}$ (DMF); IR (KBr): $v \quad 3435$, 3176(2NH), 3030, 2928(CH), 1671, 1649 (2C=O), $1594(\mathrm{C}=\mathrm{N}) \mathrm{cm}^{-1} ;{ }^{1} \mathrm{H}-\mathrm{NMR}$ (DMSO-d6) $\delta 2.36(\mathrm{~s}, 3 \mathrm{H}$, $\left.\mathrm{CH}_{3}\right), 2.76\left(\mathrm{~s}, 3 \mathrm{H}, \mathrm{CH}_{3}\right), 6.97-7.73$ (m, 15H, ArH), 10.46 (s, br, 1H, D2O-exchangeable NH), 11.72 (s, br, $1 \mathrm{H}, \mathrm{D}_{2} \mathrm{O}$-exchangeable NH); MS m/z (\%) $525\left(\mathrm{M}^{+}, 7\right), 447$ (16), 334 (100), 200 (59), 77 (89). Anal. Calcd for $\mathrm{C}_{28} \mathrm{H}_{23} \mathrm{~N}_{5} \mathrm{O}_{2} \mathrm{~S}_{2}$ (525.64): C, 63.98; $\mathrm{H}, 4.41 ; \mathrm{N}, 13.32$. Found $\mathrm{C}, 63.84 ; \mathrm{H}, 4.30 ; \mathrm{N}, 13.28 \%$.

3.2.7.3. $\quad N^{\prime}$-(3,4-Diphenylthiazol-2(3H)-ylidene)-4-methyl-2-phenylthiazole-5-carbohydrazide(27). Yellow solid (70\%); m.p. 174-178 ${ }^{\circ} \mathrm{C}(\mathrm{EtOH})$; IR (KBr) v 3369(NH), 3047, 2926(CH), $1648(\mathrm{C}=\mathrm{O}), 1594$ $(\mathrm{C}=\mathrm{N}) \mathrm{cm}^{-1} ;{ }^{1} \mathrm{H}-\mathrm{NMR}\left(\mathrm{DMSO}-d_{6}\right) \delta 2.75\left(\mathrm{~s}, 3 \mathrm{H}, \mathrm{CH}_{3}\right), 7.03(\mathrm{~s}, 1 \mathrm{H}$, thiazole-H5), 7.35-8.02 (m, 15H, ArH), 10.73 (s, br, 1H, D2O-exchangeable NH); MS m/z (\%) 468 (M+, 25), 334 (100), 200 (40), 104 (69), 64(65). Anal.Calcd for $\mathrm{C}_{26} \mathrm{H}_{20} \mathrm{~N}_{4} \mathrm{OS}_{2}$ (468.59): C, 66.64; H, 4.30; N, 11.96. Found C, 66.55; H, 4.21; N, $11.79 \%$. 
3.2.7.4. 4-Methyl- $N^{\prime}$-(4-oxo-3-phenylthiazolidin-2-ylidene)-2-phenylthiazole-5-carbohydrazide (29). Yellowish-white solid (72\%); m.p. 192-194 C (Dioxane); IR (KBr) $v$ 3331(NH), 3036, 2926 (CH), 1726, $1648(2 \mathrm{C}=\mathrm{O}), 1596(\mathrm{C}=\mathrm{N}) \mathrm{cm}^{-1} ;{ }^{1} \mathrm{H}-\mathrm{NMR}$ (DMSO-d6) $\delta 2.65\left(\mathrm{~s}, 3 \mathrm{H}, \mathrm{CH}_{3}\right), 4.23(\mathrm{~s}, 2 \mathrm{H}$, thiazolone- $\left.\mathrm{CH}_{2}\right), 7.40-7.88(\mathrm{~m}, 10 \mathrm{H}, \mathrm{ArH}), 10.82$ (s, br, 1H, $\mathrm{D}_{2} \mathrm{O}-$ exchangeable $\left.\mathrm{NH}\right) ; \mathrm{MS} m / z(\%) 408$ $\left(\mathrm{M}^{+}, 65\right), 334$ (18), 202 (100), 104 (86), 64 (69). Anal. Calcd for $\mathrm{C}_{20} \mathrm{H}_{16} \mathrm{~N}_{4} \mathrm{O}_{2} \mathrm{~S}_{2}$ (408.50): C, 58.80; H, 3.95; N, 13.72. Found C, 58.68; H, 3.84; N, 13.64\%.

\subsection{Evaluation of the antitumor activity using Viability assay:}

Human hepatocellular carcinoma (HepG2) cell line was obtained from the American Type Culture Collection (ATCC, Rockville, MD). The cells were grown on RPMI-1640 medium supplemented with $10 \%$ inactivated fetal calf serum and $50 \mu \mathrm{g} / \mathrm{mL}$ gentamycin. The cells were maintained at $37{ }^{\circ} \mathrm{C}$ in a humidified atmosphere with 5\% carbon dioxide and were subcultured 2 to 3 times a week. Potential cytotoxicity of the tested compounds was evaluated on tumor cells using the reported method of Gangadevi and Muthumary [43]. The cells were grown as monolayers in growth RPMI-1640. The monolayers of $10^{4}$ cells adhered at the bottom of the wells in a 96-well microtiter plate incubated for $24 \mathrm{~h}$ at $37{ }^{\circ} \mathrm{C}$ in a humidified incubator with $5 \%$ carbon dioxide. The monolayers were then washed with sterile phosphate buffered saline $(0.01 \mathrm{M} \mathrm{pH} \mathrm{7.2)}$ and simultaneously the cells were treated with $100 \mu \mathrm{L}$ from different dilutions of tested sample in fresh maintenance medium and incubated at $37{ }^{\circ} \mathrm{C}$. A control of untreated cells was made in the absence of tested sample. Positive controls containing doxroubcin drug was also tested as reference drug for comparison. Six wells were used for each concentration of the test sample. Every $24 \mathrm{~h}$ the observation under the inverted microscope was made.The number of the surviving cells was determined by staining the cells with crystal violet [44] followed by cell lysing using 33\% glacial acetic acid and read the absorbance at $590 \mathrm{~nm}$ using microplate reader (SunRise, TECAN, Inc, USA) after well mixing. The absorbance values from untreated cells were considered as $100 \%$ proliferation. The number of viable cells was determined using microplate reader as previously mentioned before and the percentage of viability was calculated as [1-(ODt/ODc)] x 100\% where ODt is the mean optical density of wells treated with the tested sample and ODc is the mean optical density of untreated cells. The relation between surviving cells and drug concentration is plotted to get the survival curve of each tumor cell line after treatment with the specified compound. The 50\% inhibitory concentration ( $\mathrm{IC}_{50}$ ), the concentration required to cause toxic effects in $50 \%$ of intact cells, was estimated from graphic plots.

\section{Conclusions}

In this context, a series of novel thiazoles and 1,3,4-thiadiazoles bearing thiazole were synthesized. The structure of the newly prepared compounds was established based on both elemental analysis and spectroscopic data and by an alternative method wherever possible. Moreover, the mechanisms of formation of the title compounds were discussed. Some of the synthesized compounds were evaluated for their anti-cancer activity against the human hepatocellular carcinoma (HepG2) cell line. The results showed that the thiadiazole derivatives 12d, 12c, 6g, 18b, 6c and $\mathbf{6 f}$ having $\mathrm{IC}_{50}$ values $0.82,0.91,1.06,1.25,1.29$ and $1.88 \mu \mathrm{M}$, respectively, were found to be the highly active compounds of the prepared series. Based on the experimental results of the antitumor activity, the structure-activity relationships were discussed.

Acknowledgments: The The authors extend their sincere appreciation to the Deanship of Scientific Research at the King Saud University for its funding this Prolific Research group (PRG-1437-29).

Author Contributions: S.M.G. designed research; S.M.G., M.R.A. and N.A.K. performed research and analyzed the data; S.M.G., N.A.K. and Y.N.M. wrote and approved the final manuscript.

Conflicts of Interest: The authors declare that there is no conflict of interests regarding the publication of this paper. 


\section{References}

1. Vijayaraghavalu, S.; Peetla, C.; Lu, S.; Labhasetwar, V. Epigenetic modulation of the biophysical properties of drug-resistant cell lipids to restore drug transport and endocytic functions. Mol. Pharm. 2012, 9, 2730-2742.

2. Jemal, A.; Bray, F.; Center, M.M.; Ferlay, J.; Ward, E.; Forman, D. Global cancer statistics. CA Cancer J. Clin. 2011, 61, 69-90.

3. Ferlay, J.; Shin, H.-R.; Bray, F.; Forman, D.; Mathers, C.; Parkin, D.M. Estimates of worldwide burden of cancer in 2008: GLOBOCAN 2008. Int. J. Cancer. 2010, 127, 2893-2917.

4. Patt, W.C. ; Hamilton, H.W.; Taylor, M.D.; Ryan, M.J.; Taylor Jr., D.G.; Connolly, C.J.C.; Doherty, A.M.; Klutchko, S.R.; Sircar, I.; Steinbaugh, B.A.; Batley, B.L.; Painchaud, C.A.; Rapundalo, S.T.; Michniewicz, B.M.; Olson, S.C.J. Structure-activity relationships of a series of 2-amino-4-thiazole-containing renin inhibitors.J. Med. Chem. 1992, 35, 2562-2572.

5. Gomha, S.M.; Abdel-Aziz, H.A. Synthesis of new heterocycles derived from 3-(3-methyl-1Hindol-2-yl)-3-oxopropanenitrile as potent antifungal agents. Bull. Korean Chem. Soc. 2012, 33, 2985-2990.

6. Karegoudar, P.; Karthikeyan, M.S.; Prasad, D.J.; Mahalinga, M.; Holla, B.S.; Kumari, N.S. Synthesis of some novel 2,4-disubstituted thiazoles as possible antimicrobial agents. Eur. J. Med. Chem. 2008, 43, 261-267.

7. Cukurovali, A.; Yilmaz, I.; Gur, S.; Kazaz, C. Synthesis, antibacterial and antifungal activity of some new thiazolylhydrazone derivatives containing 3-substituted cyclobutane ring. Eur. J. Med. Chem. 2006, 41, 201-207.

8. Sharma, P.K.; Sawhney, S.N.; Gupta, A.; Singh, G.B.; Bani, S.Synthesis and antiinflammatory activity of some 3-(2-thiazolyl)-1,2-benzisothiazoles. Indian J. Chem 1998, 37B, 376-381.

9. Shih, M.H.; Ying, K.F. Syntheses and evaluation of antioxidant activity of sydnonyl substituted thiazolidinone and thiazoline derivatives. Bioorg. Med. Chem. 2004, 12, 4633-4643.

10. Shiradkar, M.; Kumar, G.V.S.; Dasari,V.; Tatikonda, S.; Akula, K.C.; Shah, R. Clubbed triazoles: A novel approach to antitubercular drugs. Eur. J. Med. Chem. 2007, 42, 807-816.

11. Gomha, S.M.; Khalil, K.D.A. convenient ultrasound-promoted synthesis and cytotoxic activity of some new thiazole derivatives bearing a coumarin nucleus.Molecules. 2012, 17, 9335-9347.

12. Gomha, S.M.; Salah, T.A.; Abdelhamid, A.O. Synthesis, characterization and pharmacological evaluation of some novel thiadiazoles and thiazoles incorporating pyrazole moiety as potent anticancer agents. Monatsh. Chem. 2015, 146, 149-158.

13. Gomha, S.M.; Riyadh, S.M.; Abbas, I.M.; Bauomi, M.A. Synthetic utility of ethylidene thiosemicarbazide: Synthesis and anti-cancer activity of 1,3-thiazines and thiazoles with imidazole moiety. Heterocycles 2013, 87, 341-356.

14. Gomha, S.M.; Salah, T.A.; Hassaneen, H.M.E.; Abdel-aziz, H.; Khedr, M.A. Synthesis, characterization and molecular docking of novel bioactive thiazolyl-thiazole derivatives as promising cytotoxic antitumor drug. Molecules 2016, 21, 1-17.

15. Kushwaha, N.; Kushwaha, S.K.S.; Rai, A.K. Biological Activities of Thiadiazole Derivatives: A Review. Inter. J. Chem. Res. 2012, 4, 517-531.

16. Singh, A.k.; Mishra, G.; Jyoti, K. Review on Biological Activities of 1,3,4-Thiadiazole Derivatives. J. Applied Pharmaceutical Science 2011, 1, 44-49

17. Siddiqui, N.; Ahuja, P.; Ahsan, W.; Pandeya, S.N.; Alam, M.S. Thiadiazoles: Progress Report on Biological Activities. J. Chem. Pharmaceutical Res. 2009, 1, 19-30.

18. Gomha, S.M.; Riyadh S.M. Synthesis under microwave irradiation of $[1,2,4]$ triazolo[3,4- $b][1,3,4]$ thiadiazoles and other diazoles bearing indole moieties and their antimicrobial evaluation. Molecules 2011, 16, 8244-8256.

19. Gomha, S.M.; Ahmed, S.A.; Abdelhamid, A.O.; Synthesis and cytotoxicity evaluation of some novel thiazoles, thiadiazoles, and pyrido[2,3- $d][1,2,4]$ triazolo[4,3-a]pyrimidin-5(1H)-one incorporating triazole moiety. Molecules 2015, 20, 1357-1376.

20. Gomha, S.M.; Khalil, K.D.; El-Zanate, A.M.; Riyadh, S.M. A facile green synthesis and anti-cancer activity of bis-arylhydrazononitriles, triazolo[5,1-c][1,2,4]triazine, and 1,3,4-thiadiazoline. Heterocycles 2013, 87, 1109-1120. 
21. Gomha, S.M.; Badrey, M.G.; Edrees,M.M.Heterocyclisation of 2,5-diacetyl-3,4-disubstituted-thieno [2,3-b]thiophenebis-thiosemicarbazones leading to bis-thiazoles and bis-1,3,4-thiadiazoles as anti-breast cancer agents, J. Chem. Res. 2016, 40, 120-125.

22. Zhang, L.J.; Yang, M.Y.; Sun, Z.H.; Tan, C.X.; Weng, J.Q.; Wu, H.K.; Liu, X.H. Synthesis and Antifungal Activity of 1,3,4-Thiadiazole Derivatives Containing Pyridine Group. Lett. Drug Des. Discov. 2014, 11, 1107-1111.

23. Farag, A.M.; Kheder, N.A.; Mabkhot, Y.M. synthesis and antimicrobial evaluation of new pyrazole, thiophene, thiazole and 1,3,4-thiadiazole derivatives incorporating pyrimidine ring. Heterocycles 2009, 78, 1787-1798.

24. Kheder, N.A.; Mabkhot, Y.N.; Farag, A.M. synthesis and antimicrobial evaluation of some bis(thioxopyridine), bis(pyrazolo[3,4-b]pyridine), bis(thieno[2,3-b]pyridine), bis(1,3,4-thiadiazole) and bis-thiophene derivatives. Heterocycles 2008, 75, 2937-2948.

25. Shawali, A.S. 1,3,4-Thiadiazoles of pharmacological interest: Recent trends in their synthesis via tandem 1,3-dipolar cycloaddition. J. Adv. Res. 2014, 5, 1-17.

26. Gomha. S.M. A facile one-pot synthesis of6,7,8,9-tetrahydrobenzo[4,5]thieno[2,3-d]-1,2,4triazolo[4,5-a]pyrimidin-5-ones. Monatsh. Chem. 2009, 140, 213-220.

27. Gomha, S.M.; Eldebss,T.M.A.; Abdulla, M.M.; Mayhoub, A.S. Diphenylpyrroles: Novel p53 Activators. Eur. J. Med. Chem. 2014, 82, 472-479.

28. Gomha, S.M.; Badrey, M.G.; Abdalla, M.M.; Arafa, R.K.Novel anti-HIV-1 NNRTIs based on a pyrazolo[4,3-d]isoxazole backbone scaffold: design, synthesis and insights into the molecular basis of action, Med. Chem. Commun. 2014, 5, 1685-1692.

29. Eldebss, T.M.A.; Gomha, S.M.; Abdulla, M.M.; Arafa, R.K. Regioselective Synthesis of novel substituted pyrrole with Protein kinases inhibitor activates selectively for VEGFR-2, EGFR, PKA and CHK1. Med. Chem. Com. 2015, 6, 977-987.

30. Gomha, S.M; Eldebss, T.M.; Badrey, M.G.; Abdulla, M.M.; Mayhoub, A.S. Novel 4-Heteroaryl-Antipyrines as DPP-IV Inhibitors. Chem. Biol. Drug Des. 2015, 86, 1292-303.

31. Mabkhot, Y.N.; Alatibi, F.; El-Sayed, N.N.; Al-Showiman, S.; Kheder, N.A., Wadood, A.; Rauf, A.; Bawazeer, S.; Hadda, T. Antimicrobial Activity of Some Novel Armed Thiophene Derivatives and Petra/Osiris/Molinspiration (POM) Analyses. Molecules 2016, 21, 222.

32. Kheder, N.A. Hydrazonoyl Chlorides as Precursors for Synthesis of Novel Bis-Pyrrole Derivatives.Molecules 2016, 21, 326

33. Mabkhot, Y.N.; Kheder, N.A.; Farag, A.M. Synthesis and Antimicrobial Activity of Some New Thieno[2,3-b]thiophene Derivatives. Molecules 2013, 18, 4669-4678.

34. Kheder, N.A.; Mabkhoot, Y.N. Synthesis and antimicrobial studies of some novel bis-[1,3,4]thiadiazole and bis-thiazole pendant to thieno[2,3-b]thiophene moiety. Int. J. Mol. Sci. 2012, 13, 3661-3670.

35. Tiperciuc, B.; Zaharia, V.; Colosi, I.; Moldovan, C.; Crişan, O.; Pîrnau, A.; Vlase, L.; Duma, M.; Oniga, O.Synthesis andevaluation of antimicrobial activity of some new hetaryl-azoles derivatives obtained from 2-aryl-4- methylthiazol-5-carbohydrazides and isonicotinic acid hydrazide. J. Heterocycl. Chem. 2012, 49, 1407-1414.

36. Shawali, S.; Gomha, S.M.A new entry for short and regioselective synthesis of [1,2,4]triazolo[4,3-b][1,2,4]-triazin-7(1H)-one. .Adv. Synth. Catal. 2000, 342, 599-604, and references cited in it.

37. Abdelhamid, A.O.; Zohdi, H.F.; Rateb, N.M. Reactions with hydrazonoyl halides XXI: Reinvestigation of the reactions of hydrazonoyl bromides with 1,1-dicyanothioacetanilide. J. Chem. Res. 1998, 184-185.

38. Qiu, X.L.;Li, G.; Wu, G.; Zhu, J.; Zhou, L.; Chen, P.L.; Chamberlin, A.R.; Lee, W.H.Synthesis and biological evaluation of a series of novel inhibitor of Nek2/Hec1 analogues. J. Med. Chem. 2009, 52, 1757-1767.

39. Tsoua, H.; MacEwan, G.; Birnberg, G.; Grosu, G.; Bursavich, M.G.; Bard, J.; Brooijmansa, N.; Toral-Barzab, L.; Hollanderb, I.; Mansoura, T.S.; Ayral-Kaloustiana, S.; Yub, K.Discovery and optimization of 2-(4-substituted-pyrrolo[2,3-b]pyridin-3-yl)methylene-4-hydroxybenzofuran- 3(2H)-ones as potent and selective ATP-competitive inhibitors of the mammalian target of rapamycin (mTOR). Bioorg. Med. Chem. Lett. 2010, 20, 2321-2325. 
40. Mavrova, A.T.; Wesselinova, D.; Tsenov, Y.A.; Denkova,P.Synthesis, cytotoxicity and effects of some1,2,4-triazole and 1,3,4-thiadiazole derivatives on immunocompetent cells. Eur. J. Med. Chem. 2009, 44, 63-69.

41. Oleson, J.J.; Slobada, A.; Troy, W.P.; Halliday, S.L.; Landes, M.J.; Angier, R.B.; Semb, J.; Cyr, K.; Williams, J.H.the carcinostatic activity of some 2-amino-1,3,4-thiadiazoles. J. Am. Chem. Soc. 1955, 77, 6713-6714.

42. Matysiak, J.; Opolski.A.Synthesis and antiproliferative activity of N-substituted 2-amino-5-(2,4dihydroxyphenyl)-1,3,4-thiadiazoles. Bioorg. Med. Chem. 2006, 14, 4483-4489.

43. Gangadevi, V.; Muthumary, Preliminary studies on cytotoxic effect of fungal taxol on cancer cell lines. $J$ .African J. Biotech. 2007, 6, 1382-1686.

44. Mosmann, T. Rapid colorimetric assay for cellular growth and survival: Application to proliferation and cytotoxicity assays. J. Immunol. Methods 1983, 65, 55-63.

Sample Availability: Samples of the compounds are available from the authors.

(C) 2016 by the authors; licensee Preprints, Basel, Switzerland. This article is an open access article distributed under the terms and conditions of the Creative Commons by Attribution (CC-BY) license (http://creativecommons.org/licenses/by/4.0/). 